\section{MS1-O2 Enzyme dynamics visualized by SAXS}

David Albesa-Jové ${ }^{1}$, Marceo E. Guerin ${ }^{1}$

1. Structural Biology Unit - CIC bioGUNE, Derio, Spain; IKERBASQUE, Basque Foundation for Science, Bilbao, Spain

email: davidalbesa@gmail.com

Substrate specificity is a fundamental property of enzyme catalysis. Enzymes are characterized by their exceptional capacity to efficiently catalyze a great number of stereospecific chemical reactions in all organisms. A selective binding and stabilization of the transition state rather than more stable forms of substrates seems to be the major determinant of the chemical reaction step. To achieve such enzyme-transition state complex, a particular spatial arrangement of the active site is required, highlighting the importance of protein dynamics and conformational changes in substrate recognition and catalysis [1]. Specifically, protein conformational changes not only involve local reorganization of flexible loops and side-chain residues, but also, in many cases, domain motions and protein oligomerization events. In addition, as a consequence of protein-protein interactions, post-translational modifications, and non-covalent associations with small-molecule inhibitors or activators, protein dynamics critically modulate enzyme catalysis. Thus, the elucidations of the molecular mechanisms by which these events modulate the function and substrate specificity of enzymes represent a major challenge.

Combining Crystallography with Small-Angle X-ray Scattering (SAXS) can advance our understanding of these dynamic processes. During this talk we will focus on two cases where combining these techniques have proven important. The first, is the phosphatidylinositol mannosyltransferase PimA, an essential membrane-associated enzyme that initiates the biosynthetic pathway of key structural elements and virulence factors of the cell wall in Mycobacterium tuberculosis $[2,3]$. PimA shows an exceptional flexibility along the catalytic cycle, including $\beta$-strand-to- $\alpha$-helix and $\alpha$-helix-to- $\beta$-strand transitions [4]. The second case is the Rv2466c, a key oxidoreductase where the redox state regulates the enzyme conformation, mediating the reductive activation of TP053, a thienopyrimidine derivative that kills replicating and non-replicating Mycobacterium tuberculosis [5,6].

1. Khersonsky O, Tawfik DS. Annu. Rev. Biochem. 2010, 79:471-505.

2. Guerin ME, et al. J. Biol. Chem. 2010, 285:33577-33583.

3. Albesa-Jove D, et al. Glycobiology 2014, 24:108-124.

4. Giganti D, et al. Nat. Chem. Biol. 2014, 11:16-18.

5. Albesa-Jove D, et al. ACS Chem. Biol. 2014, 9:1567-1575.

6. Albesa-Jove D, et al. J. Biol. Chem. 2015, 290:31077-31089.

Keywords: Enzyme dynamics
MS1-O3 From size exclusion to HIS-tags: Increasing sample purity for BioSAXS

Martha E. Brennich ${ }^{1}$, Stephanie Hutin ${ }^{2}$, Katharina Weinhäupl ${ }^{2}$, Paul Schanda ${ }^{2}$, Benoit Maillot ${ }^{1}$, Petra Pernot ${ }^{1}$, Adam Round $^{3}$

1. ESRF-The European Synchrotron, Grenoble, France

2. Institute de Biologie Structurale, Grenoble, France

3. EMBL, Grenoble Outstation, France

email: brennich@esrf.fr

Small Angle X-ray scattering of proteins and nucleic acids (BioSAXS) is continuously gaining importance for structural biology. This has led to the development of dedicated, highly automated beamlines such as BM29 at the ESRF which provides a completely in vacuo setup with a flow-through capillary for samples and a sample changer robot for sample delivery.

For many macromolecules of interest, obtaining useful results is still challenging as complexes might disassociate or aggregates might form between purification and SAXS measurement, especially if buffer adjustments or transportation (including freezing and de-freezing) are required. Therefore, online size exclusion chromatography (SEC) directly at the beamline has by now become a standard approach to BioSAXS measurements. These experiments provide SEC-SAXS chromatograms that do not only allow us to obtain SAXS curves for different oligomeric species in a sample, but can also indicate conformational changes within a single species.

But in some cases, size exclusion is not the best approach as final purification step, as it requires high initial sample concentrations and fails to separate similarly sized molecules. In this presentation we show the combination of gradient-varying liquid chromatography techniques with SAXS: We use online nickel affinity chromatography to separate a complex of a chaperone protein with its target from the target and the chaperone protein alone, and ion exchange chromatography to purify and simultaneously concentrate a viral helicase complex while maintaining its monomeric state and moderate salt concentrations. Both these techniques demand careful considerations of the background correction, which will also be addressed in the talk.

Keywords: SAXS, liquid chromatography 